\title{
Analytical Solutions of the Fisher-KPP equations
}

\author{
Weishi Yin ${ }^{1,}$, Zhixia Jiang ${ }^{1, b^{*}}$ \\ ${ }^{1}$ School of Science, Changchun university of Science and Technology, Jilin 130022, P.R.China. \\ ayinweishi@foxmail.com, bhixia_jiang@126.com
}

Keywords: Traveling wave solutions; Tanh-function method; Fisher-KPP equations..

\begin{abstract}
This paper is concerned with the existence of exact traveling wave solutions of nonlinear evolution equations by the tanh-function method.The validity and reliability of this method is demonstrated by applying it to the Fisher-KPP equations.
\end{abstract}

\section{Introduction}

It is well known that nonlinear phenomena are very important in a variety of scientific fields, especially in fluid mechanics, solid state physics, plasma physics, plasma waves, capillary-gravity waves, and chemical physics. Most of these phenomena are described by nonlinear partial differential equations.Analytical solutions of this problems are usually not available, especially when the nonlinear terms are involved.Therefore, finding its travelling solutions is of practical importance.

The methods of looking for exact traveling wave solutions of nonlinear evolution equations, has been tremendous development in recent decades, such as inverse scattering method[1], Hirota's bilinear technique[2], the Painlve expansion method[7]. In the early nineties of last century, Huibin and Kelin[3]proposed a new method. The main idea of this method is taking hyperbolic tangent function of the power series as possible traveling wave solutions of the nonlinear evolution equations. Then they substituted the power series directly to KdV equation, and obtained the coefficients of the power series. However this method involved very complicated algebra computation. In order to reduce the complex algebra computation, Malfiety [4-6] proposed the tanh-function method. Since all the derivatives of hyperbolic tangent can be expressed by the hyperbolic tangent in itself, this simple translation makes the method can be applied to more nonlinear evolution equations[8-9].

\section{The Tanh-function Method}

Let' s consider the nonlinear partial differential equations

$$
N\left(u, u_{t}, u_{x}, u_{x x}, u_{x x x} \cdots\right)=0
$$

Where $u(x, t)$ is the real function on $R^{2}$. At first, we assume the traveling wave solutions of (2.1) are the form of

$$
u(x, t)=U(\omega)=U(c(x-v t))
$$

with the velocity $v$, and the constant $c$. Submitted (2.2) into (2.1), we can get the ODEs about $\omega$ !

$$
N\left(U, U^{\prime}, U^{\prime \prime}, U^{\prime \prime \prime}, \ldots\right)=0
$$

Second, we assume the possibly traveling wave solutions can be written

$$
u(x, t)=U(\omega)=H(Y)=\sum_{i=0}^{K} a_{i} Y^{i}
$$

where $Y=\tanh (\omega)=\frac{e^{\omega}-e^{-\omega}}{e^{\omega}+e^{-\omega}}$, the highest order $K$ will be determined late. Then we can get 


$$
\begin{aligned}
& \frac{d Y}{d \omega}=1-Y^{2}, \frac{d U}{d \omega}=\left(1-Y^{2}\right) H^{\prime}, \frac{d^{2} U}{d \omega}=\left(1-Y^{2}\right)\left(-2 Y \cdot H^{\prime}+\left(1-Y^{2}\right) H^{\prime \prime}\right) \\
& \frac{d^{3} U}{d \omega^{3}}=\left(1-Y^{2}\right)\left(6 Y^{2}-2\right) H^{\prime}-6 Y\left(1-Y^{2}\right) H^{\prime \prime}+\left(1-Y^{2}\right)^{2} H^{\prime \prime \prime}
\end{aligned}
$$

Submitted above equations into (2.3), we can get the ODEs with $Y$

$$
N\left(Y, H, H^{\prime}, H^{\prime \prime}, H^{\prime \prime \prime}, \ldots\right)=0
$$

where $H^{\prime}=\frac{d H}{d Y}$ : To determine the parameter $K$, we usually balance the nonlinear term and the highest order derivative term in equation (2.5). Then, we submitted (2.4) (with the determined $K$ ) into (2.5), and get the polynomial equation with $Y$ : Collecting all the coefficients of power of $Y$; and letting the coefficients of each power of $Y$ to be vanished, we can determined all the coefficient $a_{1}, a_{2}, \ldots, a_{K}$. According to (2.4), we can get the traveling wave solutions of (2.1).

\section{Application}

Let us consider the Fisher-KPP equations

$$
u_{t}=u_{x x}+b u-a u^{3}
$$

where $a, b$ are non-negative constants. Submitting (2.2) and (2.4) into (3.1), we can get

$$
-c v\left(1-Y^{2}\right) H^{\prime}=c^{2}\left(1-Y^{2}\right)\left[-2 Y \cdot H^{\prime}+\left(1-Y^{2}\right) H^{\prime \prime}\right]+b H-a H^{3}
$$

Balancing $Y^{4} H^{\prime \prime}$ with $H^{3}$; which yields $K=1$, So, we can get

$$
H(Y)=a_{0}+a_{1} Y
$$

Substituting $H(Y), H^{\prime}(Y)$ and $H^{\prime \prime}(Y)$ into above equation yields algebraic equations of $a_{0}, a_{1}, c$ and $v$ :

$$
\left\{\begin{array}{c}
b a_{0}-a a_{0}^{3}+a_{1} c v=0 \\
-2 a_{1} c^{2}+b a_{1}-3 a a_{0}^{2} a_{1}=0 \\
-a_{1} c v-3 a a_{0} a_{1}^{2}=0 \\
2 a_{1} c^{2}-a a_{1}^{3}=0
\end{array}\right.
$$

Solving the algebraic equations, we obtain

$$
\begin{aligned}
& c=\frac{\sqrt{2 b}}{2}, v=0, a_{0}=0, a_{1}=-\frac{\sqrt{a b}}{a} ; \\
& c=-\frac{\sqrt{2 b}}{2}, v=0, a_{0}=0, a_{1}=\frac{\sqrt{a b}}{a} ; \\
& c=\frac{\sqrt{2 b}}{4}, v=-\frac{3 \sqrt{2 b}}{2}, a_{0}=\frac{\sqrt{a b}}{2 a} a_{1}=\frac{\sqrt{a b}}{2 a} ; \\
& c=-\frac{\sqrt{2 b}}{4}, v=-\frac{3 \sqrt{2 b}}{2}, a_{0}=-\frac{\sqrt{a b}}{2 a} a_{1}=\frac{\sqrt{a b}}{2 a} ; \\
& c=\frac{\sqrt{2 b}}{4}, v=\frac{3 \sqrt{2 b}}{2}, a_{0}=\frac{\sqrt{a b}}{2 a} a_{1}=-\frac{\sqrt{a b}}{2 a} ; \\
& c=\frac{\sqrt{2 b}}{4}, v=\frac{3 \sqrt{2 b}}{2}, a_{0}=-\frac{\sqrt{a b}}{2 a} a_{1}=\frac{\sqrt{a b}}{2 a} ;
\end{aligned}
$$


In view of (3.2) we obtain the traveling wave solution of (3.1)

$$
\begin{aligned}
& u(x, t)=-\frac{\sqrt{a b}}{a} \tanh \left(\frac{\sqrt{2 b}}{2} x\right), \\
& u(x, t)=\frac{\sqrt{a b}}{a} \tanh \left(\frac{\sqrt{2 b}}{2} x\right), \\
& u(x, t)=\frac{\sqrt{a b}}{2 a}+\frac{\sqrt{a b}}{2 a} \tanh \left[\frac{\sqrt{2 b}}{4}\left(x+\frac{3 \sqrt{2 b}}{2} t\right)\right], \\
& u(x, t)=-\frac{\sqrt{a b}}{2 a}-\frac{\sqrt{a b}}{2 a} \tanh \left[\frac{\sqrt{2 b}}{4}\left(x+\frac{3 \sqrt{2 b}}{2} t\right)\right], \\
& u(x, t)=-\frac{\sqrt{a b}}{2 a}+\frac{\sqrt{a b}}{2 a} \tanh \left[\frac{\sqrt{2 b}}{4}\left(x-\frac{3 \sqrt{2 b}}{2} t\right)\right], \\
& u(x, t)=\frac{\sqrt{a b}}{2 a}-\frac{\sqrt{a b}}{2 a} \tanh \left[\frac{\sqrt{2 b}}{4}\left(x-\frac{3 \sqrt{2 b}}{2} t\right)\right],
\end{aligned}
$$
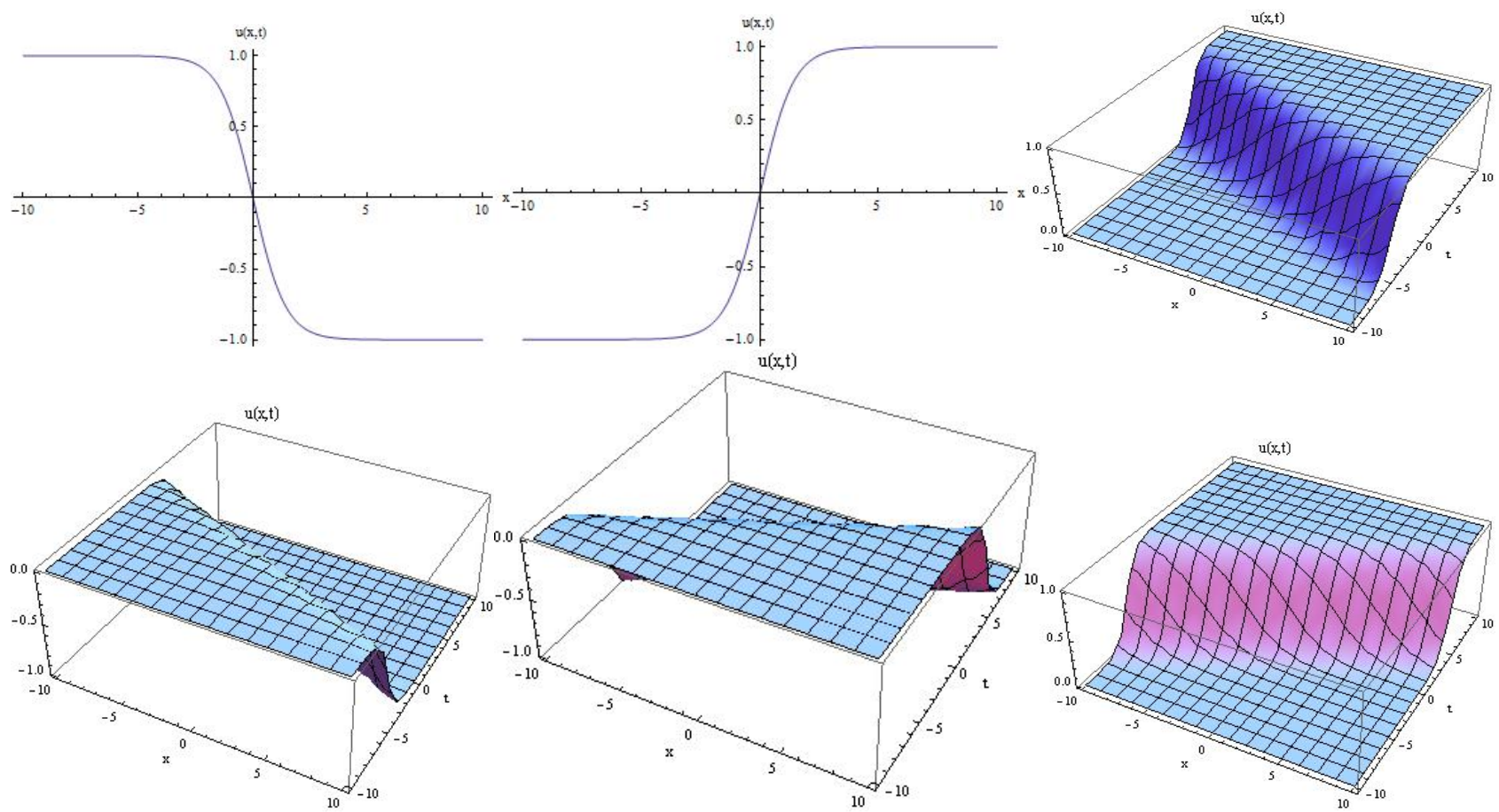

Fig. 1. the travelling wave solutions of (3.3), when we take $a=b=1$ and $(x, t) \in[-10,10] \times[-10,10]$.

In the similar way, we can also get the following solutions of $(3.1)$

$$
\begin{aligned}
& u(x, t)=-\frac{\sqrt{a b}}{a} \operatorname{coth}\left(\frac{\sqrt{2 b}}{2} x\right), \\
& u(x, t)=\frac{\sqrt{a b}}{a} \operatorname{coth}\left(\frac{\sqrt{2 b}}{2} x\right), \\
& u(x, t)=\frac{\sqrt{a b}}{2 a}+\frac{\sqrt{a b}}{2 a} \operatorname{coth}\left[\frac{\sqrt{a b}}{4}\left(x+\frac{3 \sqrt{2 b}}{2} t\right)\right], \\
& u(x, t)=-\frac{\sqrt{a b}}{2 a}-\frac{\sqrt{a b}}{2 a} \operatorname{coth}\left[\frac{\sqrt{a b}}{4}\left(x+\frac{3 \sqrt{2 b}}{2} t\right)\right], \\
& u(x, t)=-\frac{\sqrt{a b}}{2 a}+\frac{\sqrt{a b}}{2 a} \operatorname{coth}\left[\frac{\sqrt{a b}}{4}\left(x-\frac{3 \sqrt{2 b}}{2} t\right)\right],
\end{aligned}
$$




$$
u(x, t)=\frac{\sqrt{a b}}{2 a}-\frac{\sqrt{a b}}{2 a} \operatorname{coth}\left[\frac{\sqrt{2 b}}{4}\left(x-\frac{3 \sqrt{2 b}}{2} t\right)\right]
$$

\section{References}

[1] M.J. Ablowitz, P.A. Clarkson, Solitons, Nonlinear Evolution and Inverse Scattering,Cambridge Univ. Press, 1991.

[2] C.H. Gu, Soliton Theory and Its Application, Springer, Berlin, 1995.

[3] L. Huibin, W. Kelin, Exact solutions for two nonlinear equations: I, J. Phys. A: Math. Gen. 23(1990), 3923-3928.

[4] W. Malfliet, Solitary wave solutions of nonlinear wave equations, Am. J. Phys. 60 (7),(1992), 650-654.

[5] W. Malfliet, The tanh method: I. Exact solutions of nonlinear evolution and wave equations, Physica Scripta, 54 (1996), 563-568.

[6] W. Malfliet, The tanh method: II. Perturbation technique for conservative systems, Physica Scripta, 54 (1996), 569-575.

[7] B.Tian, Y. Gao, Truncated Painleve expansion and a wide-ranging type of generalized variable-coefficient Kadomtsev-Petviashvili equations, Phys. Let. A, 209 (1995), 297-304.

[8] M.A. Abdou, New exact travelling wave solutions using modified extended tanh-function method, Chaos, Solitons and Fractals ,31 (2007), 840-85.

[9] A.H. Khater, W. Malfliet, D. K. Callebaut, E. S. Kamel, The tanh method, a simple transformation and exact analytical solutions for nonlinear reaction-diffusion equations,Chaos Soliton. Fract,14 (2002), 513-522. 